\title{
Noninvasive stress testing of myocardial perfusion defects: head-to-head comparison of thallium-201 SPECT to MRI perfusion
}

\author{
Gabriella Vincenti, MD, ${ }^{\mathrm{a}}$ René Nkoulou, $M D,{ }^{\mathrm{a}}$ Charles Steiner, $M D,{ }^{\mathrm{b}}$ \\ Hestia Imperiano, MD, ${ }^{b}$ Giuseppe Ambrosio, MD, ${ }^{d}$ François Mach, MD, ${ }^{a}$ \\ Osman Ratib, $M D,{ }^{b}$ Jean-Paul Vallee, $M D,{ }^{c}$ and Thomas H. Schindler, $M^{a}$
}

Background. To evaluate the diagnostic value of magnetic resonance imaging (MRI) of myocardial perfusion in the assessment of flow-limiting epicardial stenosis in a head-to-head comparison with abnormal thallium-201 $\left({ }^{201} \mathrm{TI}\right)$ single photon emission tomography (SPECT) studies in patients with predominantly known coronary artery disease (CAD).

Methods and Results. Twenty-one patients (mean age $65 \pm 10$ years) with reversible myocardial perfusion defects on ${ }^{201}$ TI-SPECT images during dipyridamole-stimulated hyperemia were recruited for study purpose. Within 5 days of the ${ }^{201}$ TI-SPECT study, myocardial perfusion was studied again with MRI during dipyridamole stimulation and at rest. Overall, ${ }^{201}$ TI-SPECT identified 30 reversible regional perfusion defects. The sensitivity to detect hypoperfused segments was $70 \%(21 / 30)$ with the GRE-MRI perfusion analysis with ${ }^{201}$ TI-SPECT as reference. When patients were subgrouped according to the extent of regional reversible perfusion defects on ${ }^{201}$ TI-SPECT, mild- (SDS: 2-4), moderate- (SDS: 5-8), and severe(SDS > 8) perfusion defects were also identified by GRE-MRI perfusion analysis in $75 \%(6 / 8)$, in $56 \%(9 / 16)$ and $100 \%(6 / 6)$, respectively.

Conclusions. GRE-MRI first-pass stress perfuslion imaging may not identify up to $30 \%$ of mild-to-moderate perfusion defects in a group of preselected patients with predominantly known CAD and abnormal ${ }^{201}$ TI-SPECT studies. (J Nucl Cardiol 2009;16:549-61.)

Key Words: Cardiac imaging $\cdot$ coronary artery disease $\cdot$ ischemia $\cdot$ MRI $\cdot$ SPECT

\section{INTRODUCTION}

According to the ischemic cascade, ${ }^{1}$ stress-induced reductions in regional myocardial perfusion as a consequence of flow-limiting epicardial stenosis commonly precede a reduction in systolic function. Nuclear imaging, therefore, has focused on imaging of myocardial perfusion during bicycle-exercise or pharmacologic

From the Department of Internal Medicine, ${ }^{\mathrm{a}}$ Cardiovascular Center, Nuclear Cardiology; Department of Radiology, ${ }^{b}$ Division of Nuclear Medicinel; Department of Radiology, ${ }^{\mathrm{c}}$ University Hospital of Geneva, Geneva, Switzerland; Division of Cardiology, ${ }^{\mathrm{d}}$ University Hospital of Perugia, Perugia, Italy.

Received for publication Jan 19, 2009; final revision accepted May 1, 20092, 2009.

Reprint requests: Thomas H. Schindler, MD, Department of Internal Medicine, Cardiovascular Center, Nuclear Cardiology, University Hospital of Geneva, Rue Micheli-du-Crest 24 CH-1211, Geneva, Switzerland; thomas.schindler@hcuge.ch.

$1071-3581 / \$ 34.00$

Copyright $(2009$ by the American Society of Nuclear Cardiology. doi:10.1007/s12350-009-9097-6 vasodilation and at rest for the identification of flowlimiting epicardial stenoses. Numerous studies have shown a higher sensitivity for nuclear perfusion imaging than for stress echocardiography with evaluation of regional systolic function ( $85 \%$ vs $75 \%$ ) in the detection of the CAD process, while a higher specificity was observed for stress echocardiography ( $88 \%$ vs $79 \%)^{2,3}$ More recently, cardiac magnetic resonance imaging (MRI) has emerged as a new and promising cardiac imaging modality to assess myocardial perfusion at rest and during pharmacologically induced hyperemia using gadolinium contrast agent and intravascular first-pass imaging. ${ }^{4}$ In a total of 15 studies comprising 355 patients, MR first-pass perfusion imaging during vasodilator stress revealed a mean sensitivity of $77 \%$ and specificity of $85 \% .^{5}$ In particular, MRI affords the advantage of a spatial resolution as high as $2-3 \mathrm{~mm}$ that even enables the imaging of stress-induced subendocardial flow reductions. ${ }^{6}$ Conversely, scintigraphic myocardial perfusion imaging with thallium-201 $\left({ }^{201} \mathrm{TI}\right)$ and single photon emission tomography (SPECT) 
provides relatively high contrast resolution owing to the high myocardial first-pass extraction fraction of ${ }^{201} \mathrm{TI}$ as high as $80-85 \% .^{7}$ While myocardial scintigraphy with ${ }^{201}$ TI-SPECT has a low effective spatial resolution of about $20 \mathrm{~mm}$, its high contrast resolution may allow the detection of even mild regional differences of the myocardial perfusion during bicycle-exercise stress or pharmacologically induced hyperemia. ${ }^{8-10}$ This may allow not only the identification of advanced flow-limiting epicardial lesions but also of early stages of subclinical and diffuse CAD as it has also been described for PET imaging. ${ }^{8,9,11,12}$ Currently, the diagnostic value of MR first-pass perfusion imaging in the identification of flow-limiting epicardial lesions as compared to cardiac SPECT as reference remains uncertain.

In view of marked differences in the methodology of cardiac ${ }^{201}$ TI-SPECT and MRI, we aimed to evaluate the diagnostic value of MR perfusion imaging in the assessment of flow-limiting epicardial stenosis in a head-to-head comparison with abnormal ${ }^{201}$ TI-SPECT studies in patients with predominantly known coronary artery disease (CAD).

\section{PATIENTS AND METHODS}

Twenty-one patients (mean age $65 \pm 10$ years, 20 men, 1 women) with a history of angina pectoris and predominantly known CAD were studied (Table 1). Of these patients, 14 patients had had previous myocardial infarction (Table 2). ${ }^{201}$ TI-SPECT was performed using standard protocol with dipyridamole-induced hyperemic flow increases and redistribution images after 4 hours. Only patients with ${ }^{201}$ TI-SPECT identified reversible perfusion defects were recruited for study purpose. In these patients, fast gradient-echo (GRE) magnetic resonance (MR) first-pass perfusion imaging was performed within 5 days of the ${ }^{201}$ TI-SPECT study applying the same vasomotor-stress protocol. Coronary angiography was performed within 2 weeks of cardiac perfusion imaging studies. Between cardiac perfusion imaging and coronary angiography, no clinical events were noted. Any anti-ischemic medication was stopped at least 12 hours before each study. In addition, no patient consumed beverages containing caffeine (tea, coffee, coca-cola, and chocolate) at least 24 hours before each cardiac perfusion study. None of the patients had a history of bronchial asthma and none was taking aminophylline or related medication.

\section{THALLIUM-201 MYOCARDIAL PERFUSION IMAGING}

For myocardial perfusion imaging with ${ }^{201} \mathrm{Tl}-$ SPECT, we used a standard protocol with imaging
Table 1. Patient characteristics

\begin{tabular}{ll}
\hline $\mathrm{n}$ & 21 \\
Sex, F/M & $1 / 20$ \\
Age, years & $65 \pm 10$ \\
BMI, kg/m ${ }^{2}$ & $27 \pm 3$ \\
Obesity & $8(38)$ \\
Hypertension & $14(66)$ \\
Diabetes mellitus & $7(33)$ \\
Hypercolesterolemia & $16(76)$ \\
Smoking & $16(76)$ \\
CAD in family & $9(43)$ \\
Medication & \\
$\quad$ Beta-Blockers & $15(71)$ \\
$\quad$ ACE-Inhibitor & $14(66)$ \\
$\quad$ Calcium-channel blocker & $8(38)$ \\
$\quad$ Nitrate & $4(19)$ \\
$\quad$ LVEF (\%) & $52 \pm 9$ \\
Known CAD & $19(90)$ \\
Vessel disease & \\
$\quad$ Single & $3(16)$ \\
Double & $5(26)$ \\
Triple & $11(58)$ \\
Myocardial infarction & $14(66)$ \\
\hline
\end{tabular}

Values are mean \pm SD or $n(\%)$.

$B M I$, Body mass index; $L V E F$, left-ventricular ejection fraction.

acquisition immediately after increasing coronary flow due to dipyridamole stimulation and redistribution images after 4 hours. Dipyridamole was infused over 4 minutes (total dose $0.56 \mathrm{mg} / \mathrm{kg}$ ), monitoring ECG and blood pressure every minute, followed by an injection of $1.5 \mathrm{MBq} / \mathrm{kg}$ of ${ }^{201} \mathrm{TI} 3 \mathrm{~min}$ from the end of the dipyridamole infusion. The myocardial perfusion data were acquired with SPECT using standard acquisition protocols. SPECT images were obtained with a rotating camera (CardioMD, Philips Medical Systems). Thirtytwo planar images $(64 \times 64$ pixel matrix, $400 \mathrm{~mm}$ field of view, $60 \mathrm{~s}$ per image) were acquired over a $180^{\circ}$ arc from the right anterior oblique position to the left posterior oblique position. From the transaxial tomograms, short-axis and long-axis myocardial slices were reoriented and the corresponding polar maps were submitted to visual and semiquantitative analysis. ${ }^{13}$

Before image acquisition, a 5-s transmission scan over the heart was performed to acquire a valid attenuation map. Tomographic reconstruction was performed with AutoSpect and Vantage Pro programs (Philips Medical Systems). ${ }^{13}$ All emission images were automatically corrected for nonuniformity, radioactive decay, and motion during acquisition. The noncorrected stress scintigraphic perfusion images were reconstructed by filtered back-projection with a Butterworth filter 
Table 2. Angiographic (percentage luminal narrowing) data, perfusion abnormalities on ${ }^{201} \mathrm{TI}-\mathrm{SPECT}$ and GRE-MRI

\begin{tabular}{lrrrrlll}
\hline $\begin{array}{c}\text { Patient } \\
\text { no. }\end{array}$ & LM & LAD & LCX & RCA & 201TI-SPECT & $\begin{array}{c}\text { Infarct } \\
\text { location } \\
\text { 201TI-SPECT }\end{array}$ & $\begin{array}{c}\text { Perfusion } \\
\text { abnormalities } \\
\text { abnormalities } \\
\text { MRI }\end{array}$ \\
\hline 1 & - & - & - & 100 & Lat, Inf & Inf, Sep & Inf, Sep \\
2 & - & 100 & 72 & - & Inf & Ant, Lat & - \\
3 & - & - & 61 & 100 & Inf & Inf & Inf \\
4 & - & 64 & 100 & 100 & - & Lat, Inf & Lat, Inf \\
5 & - & 57 & 100 & 100 & - & Lat, Inf & Lat, Inf \\
6 & - & - & 100 & 100 & Lat, Inf & Lat, Inf & Lat, Inf \\
7 & - & - & 100 & 100 & - & Inf & - \\
8 & - & 98 & - & 56 & Inf & Ant, Lat & - \\
9 & - & 65 & 53 & 75 & - & Inf & - \\
10 & - & 100 & - & 56 & Ant, Sep & Ant, Sep & - \\
11 & - & 100 & - & 100 & Ant, Sep, Inf & Ant, Sep, Inf & - \\
12 & - & - & - & 88 & - & Inf & - \\
13 & - & 100 & - & - & Ant & Ant, Sep & Ant, Sep \\
14 & - & 89 & - & - & Ant & Ant, Sep & Ant, Sep \\
15 & - & - & 86 & - & - & Lat, Inf & - \\
16 & - & - & 83 & - & Lat, Inf & Lat, Inf & Lat, Inf \\
17 & - & 100 & - & - & - & Ant, Sep & Ant, Sep \\
18 & - & 100 & 100 & - & Inf & Ant, Inf & Ant, Inf \\
19 & - & 100 & - & 100 & Inf & Ant, Inf & Ant, Inf \\
20 & - & 100 & - & 100 & Inf & Ant, Inf & Ant, Inf \\
21 & - & 61 & 79 & 92 & Inf & Lat, Inf & Lat, Inf \\
\hline
\end{tabular}

-, No abnormality; Ant, anterior wall; Sep, interventricular septum; Lat, lateral wall; Inf, inferior wall; $L M$, left main artery; $L A D$, left anterior descending artery; $L C X$, left circumflex artery; $R C A$, right coronary artery.

(order, 5; cutoff, 0.66). Attenuation maps, corrected for ${ }^{201}$ TI downscatter, were reconstructed by use of a Bayesian prior approach after logarithmic inversion and normalization to a reference scan and the application of a Butterworth filter (order, 5; cutoff, 0.50).

\section{MAGNETIC RESONANCE IMAGING}

A $1.5 \mathrm{~T}$ scanner (Siemens Magnetom Espree) with a phased-array receiver coil was used. Balanced steadystate free precession (SSFP) cine imaging (parallel imaging technique GRAPPA 2) with retrospective gating was acquired in vertical long axis (two chambers), horizontal long axis (four chambers), and in multiple short-axis views covering the left ventricle, for the assessment of left ventricular function. Typical parameters were repetition time (TR) $3.42 \mathrm{~ms}$, echo time (TE) $1.51 \mathrm{~ms}$, flip angle $50^{\circ}$, leading to a spatial resolution of $1.8 \times 1.3 \times 8 \mathrm{~mm}^{3}$. Subsequently, dipyridamole $(0.56 \mathrm{mg} / \mathrm{kg})$ was infused over 4 minutes monitoring ECG and blood pressure every minute. Three minutes after finishing the dipyridamole infusion (waiting peak vasodilation), bolus injection of gadolinium contrast
(0.1 mmol/kg Gadoteric acid, Dotarem, Guerbet) was performed. MR first-pass stress perfusion study was acquired in three short-axis planes (basal, middle, and apical) and in one vertical long-axis plane every one heart beat (single shot acquisition) with a saturation-recovery turbo-FLASH gradient-echo (GRE) sequence, delayed time of $90 \mathrm{~ms}$, and parallel imaging technique read out (GRAPPA 3). Typical parameters were repetition time (TR) $1.92 \mathrm{~ms}$, echo time (TE) $0.88 \mathrm{~ms}$, and flip angle $15^{\circ}$, leading to a spatial resolution of $2.9 \times 3 \times 8 \mathrm{~mm}^{3}$. Additional gadolinium $0.1 \mathrm{mmol} / \mathrm{kg}$ was injected for the delayed contrast enhancement imaging acquired after 10 minutes to allow for the clearance of the first contrast agent injection. Delayed enhancement (DE)-MRI was performed with a segmented FLASH 3D inversionrecovery technique: inversion time set to null normal myocardium. Additional PSIR 2D sequences were acquired in the vertical long axis, horizontal long axis, and short-axis planes for a more accurate analysis of the hyper-enhanced regions. After the acquisition of the first-pass stress perfusion images, $240 \mathrm{mg}$ aminophylline was administered intravenously in order to reverse the vasodilatory effects of dipyridamole and, thereby, 
to avoid lingering effects of dipyridamole on the assessment of rest perfusion following the stress protocol. Subsequently, first-pass perfusion imaging was repeated at rest using the same dose of contrast agent (0.1 mmol/kg).

\section{IMAGE ANALYSIS}

\section{Myocardial Scintigraphy}

Visual interpretation was performed on eight shortaxis planes (from apex to base), eight vertical long-axis planes and eight horizontal long-axis planes both during stress test and at rest (total of 24 slices). Segments with "reversible", perfusion defects were considered ischemic while segments with "fixed" perfusion defects during stress and at rest identified necrosis. Quantitative and observer-independent evaluation of ${ }^{201}$ Tl-SPECT was performed using a 20 -segment model. ${ }^{14}$ Each segment was scored using a 5-point scoring system $(0=$ normal, $1=$ mildly, $2=$ moderate, $3=$ severe reduction of radioisotope uptake, and $4=$ apparent absence of detectable tracer uptake in a segment). A summed stress score (SSS) was obtained by adding the scores of the 20 segments of the stress images. ${ }^{14}$ Similarly, a summed rest score (SRS) was obtained by adding the scores of the 20 segments on the rest images. For the assessment of the extent of myocardial ischemia, a summed difference score (SDS) was calculated by subtracting the SRS from the SSS. A SSS $<4$ was considered normal, 4-8 mildly abnormal, 9-13 moderately abnormal, and $>13$ severely abnormal perfusion. ${ }^{14}$ Further, a $\operatorname{SDS} \geq 2$ identified ischemia, while a SDS $<2$ was considered a normal. The extent of regional reversible perfusion defects on ${ }^{201}$ TI-SPECT was graded according the SDS value. According to this, a SDS of $\geq 2-4,>5-8$, and $>8$ defined mild, moderate, and severe reversible perfusion defects, respectively. Finally, the left-ventricular ejection fraction (LVEF) at rest and after dipyridamole stress test was evaluated by gated SPECT.

\section{MR Perfusion Imaging}

MR stress and rest perfusion images and delayed enhancement images in equivalent slices were evaluated by two independent observers according to the AHA 17-segment model. ${ }^{15}$ Two expert observers, aware of the results of the ${ }^{201}$ TI-SPECT study, independently evaluated the MR perfusion images, and in the case of disagreement between both observers, consent was achieved in a joint reading. The analysis of perfusion images was visual, based on gadolinium signal intensity on first-pass images during vasomotor stress and its difference from the images at rest. GRE-MR gadolinium first-pass stress and rest perfusion images of three shortaxis planes (basal, middle, and apical) and in one vertical long-axis plane were visually compared side-byside. Abnormal perfusion was defined as a signal reduction (myocardium being black) at the peak bolus during stress test but not at rest; normal perfusion was defined as myocardium being bright both during stress and at rest. Criteria indicative for true hypoperfusion vs artifacts were subendocardial signal intensity reduction persisting longer than the contrast-medium first-pass through the left ventricle cavity (susceptibility artifacts tend to be worst during peak concentration of gadolinium in the RV and LV cavities and tend to disappear rapidly), signal intensity reduction in several slices, perfusion defect tending to follow the typical perfusion territory of a coronary artery, absence of breathing motion, and triggering artifacts during CM first-pass. ${ }^{16}$ Ischemic segments were scored with a four-point scale: 0 , normal; 1 , probably normal; 2 , probably abnormal; 3 , definitely abnormal. This scoring system was chosen to allow dichotomization of results into normal $(\leq 1)$ and abnormal $(\geq 2)$. Hyperenhanced regions on DE-CMR were assumed to represent myocardial infarction unless isolated midwall or subepicardial hyperenhancement was found. ${ }^{17}$ Necrosis appeared hyper-enhanced compared with normal myocardium in delayed enhancement. In presence of necrosis, residual ischemia was defined as perfusion defect more extensive than the region of delayed enhancement.

\section{Coronary Angiography}

Standard coronary angiography of the left and right coronary arteries in multiple views was performed according to the Judkins technique. Angiograms were analyzed visually by two experienced observers who identified the stenotic segments. The luminal diameter of the stenosed artery was evaluated with quantitative coronary angiography (QCA) in the projection that demonstrated the most severe stenosis. Morphologically significant stenosis was defined as $\geq 50 \%$ diameter stenosis.

\section{Statistical Analysis}

Data are presented as mean \pm SD for quantitative and absolute frequencies for qualitative variables. For comparison of differences, appropriate $t$ tests for independent or paired samples were used (Statistical Analysis Software Institute, Cary, NC, USA). Differences in the agreement of myocardial perfusion defects were analyzed with the $x^{2}$ test. Two-tailed $P$ values $<0.05$ were considered to be statistically significant. 


\section{RESULTS}

\section{Clinical Characteristics}

Table 1 summarizes the characteristics of the study population. All patients had a history of angina pectoris, 19 with known CAD and two with suspicion for CAD. Of these, 14 had previous nontransmural myocardial infarction. The mean LVEF as determined by gated SPECT was $52 \pm 9 \%$. When a significant epicardial coronary artery lesion during coronary angiography was defined as $\geq 50 \%$ diameter stenosis, 11 patients had three vessel disease, 5 two vessel disease, and 3 one vessel disease. Most patients had hypercholesterolemia and smoking, followed by arterial hypertension, family history of CAD, type 2 diabetes mellitus, and obesity as defined by body mass index $\geq 30 \mathrm{~kg} / \mathrm{m}^{2}$.

\section{Myocardial Perfusion Imaging}

Figure 1 illustrates an example of a myocardial perfusion defect in the antero-septo-apical wall during dipyridamole stimulation on polar maps and threedimensional (3D) ${ }^{201}$ TI-SPECT images in a 64-year-old and asymptomatic diabetic patient (Fig. 1A) as well as on a mid-ventricular short-axis plane on GRE-MR firstpass imaging (Fig. 1B). The stress-induced regional perfusion defect was related to high grade epicardial lesions in the proximal and middle artery segment of the left-anterior descending artery (LAD) as shown on coronary angiography (Fig. 1C).

Conversely, Fig. 2 demonstrates an example of a 42-year-old chronic smoker, who had had an acute coronary syndrome (ACS) 5 days before the imaging study. Coronary angiography demonstrated a $50 \%$ stenosis of the proximal right coronary artery (RCA) as well as a $90 \%$ stenosis of posterior descending artery (PDA) (Fig. 2A). Subsequently, a cardiac ${ }^{201}$ TI-SPECT study was performed to evaluate the hemodynamic significance of these stenoses. Figure 2B depicts a stress-induced myocardial perfusion defect in the inferior wall on polar maps and 3D ${ }^{201}$ TI-SPECT images, which was not seen on the basal and mid-ventricular short-axis plane on GRE-MR first perfusion imaging (Fig. 2C). The hemodynamic significance of the stenoses of the RCA were also evidenced by the post-stress gated SPECT identified hypokinesia in the territory of the stress-induced myocardial perfusion defect in the inferior wall not seen on resting gated SPECT, indicative of myocardial stunning.

In 21 patients predominantly with known multivessel disease, however, ${ }^{201}$ TI-SPECT with stress and redistribution images identified 30 regional reversible perfusion defects (abnormal SSS $\geq 4$ and $\mathrm{SDS} \geq 2$ )
(Tables 2 and 3). The mean SRS, SSS, and SDS were $9 \pm 8,18 \pm 9$, and $9 \pm 4$, respectively. All reversible perfusion defects on ${ }^{201}$ TI-SPECT were subtended to epicardial lesions $>75 \%$ diameter stenosis as evidenced by QCA (Table 2). Reversible perfusion defects of the inferior and infero-lateral wall were confirmed on attenuation-corrected ${ }^{201}$ TI-SPECT images (Table 2). Coronary artery lesions for these reversible perfusion defects ranged from $75 \%$ to $100 \%$ diameter stenosis with a mean value of $93 \pm 9 \%$. On a patient-based analysis, mild- (SDS: 2-4), moderate- (SDS: 5-8), and severe- (SDS > 8) reversible perfusion defects were observed in $14 \%(3 / 21), 47 \%(10 / 21)$, and $38 \%(8 / 21)$ on ${ }^{201}$ TI-SPECT. Conversely, on a vessel-based analysis, there were $27 \%$ (8/30) mild-, $57 \%$ (16/30) moderate, and $20 \%(6 / 30)$ severe reversible perfusion defects (Table 3). In these patients with 30 regional reversible scintigraphic perfusion defects on ${ }^{201}$ TI-SPECT, GREMRI of myocardial perfusion during dipyridamole stimulation and at rest detected 21 regional perfusion defects (Tables 2, 3, Fig. 3). As can be seen in Table 3, there was a good agreement of 70\% (21/30) of regional perfusion defects during pharmacologic vasodilation between ${ }^{201}$ TI-SPECT and GRE-MRI, while nine regional perfusion defects as defined by ${ }^{201}$ TI-SPECT were not necessarily identified on GRE-MR images. This difference achieved statistical significance $(P \leq 0.001)$. Of these ${ }^{201}$ TI-SPECT defined nine regional reversible perfusion defects, that were not identified by GRE-MRI, four were scored as "probably" normal (perfusion score of one), and five as definitively normal on GRE-MRI. The segments scored as "probably normal" on GRE-MRI corresponded to two mild and two moderate reversible perfusion defects on ${ }^{201}$ TI-SPECT, whereas the five segments scored as "definitively normal" on GRE-MRI corresponded to moderate ischemia on ${ }^{201} \mathrm{TI}$-SPECT. The disagreement between both modalities was seen myocardial segments with and without previous myocardial infarction (Tables 2 and 3). In regions with previous myocardial infarction, 5 out of 17 residual myocardial ischemia as denoted by ${ }^{201}$ TI-SPECT were not identified by GREMRI. In these 17 patients, both delayed enhancement MRI and ${ }^{201}$ TI-SPECT denoted infarcted regions. While ${ }^{201}$ TI-SPECT also identified a significant reversibility of stress-rest perfusion in the infarcted area indicative for peri-infarctial ischemia, this was not observed in five of these cases where perfusion MRI demonstrated a matched stress-rest perfusion defect corresponding to the delayed enhancement images. Conversely, in patients without previous myocardial infarction, ${ }^{201}$ TI-SPECT defined 13 reversible regional perfusion defects, of which four were not demonstrated on GRE-MR stress perfusion images. 


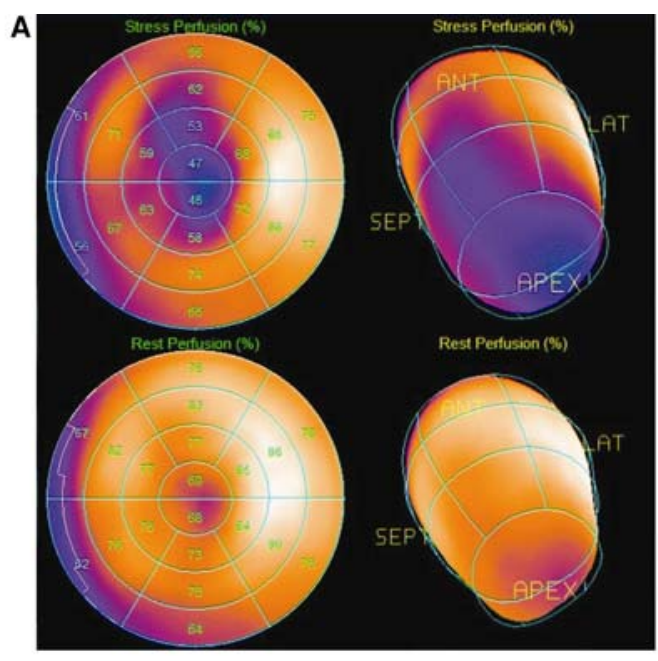

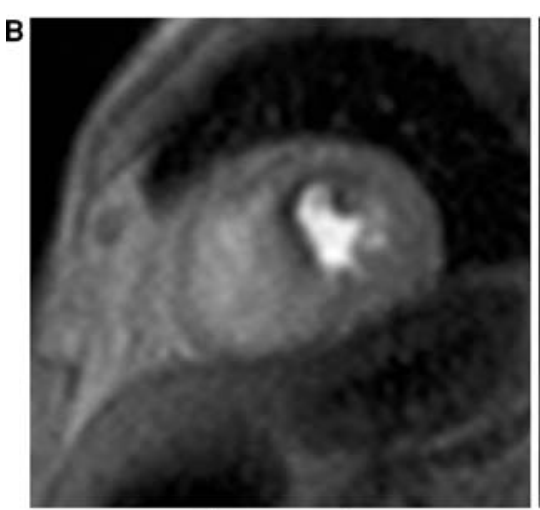

Hyperemia

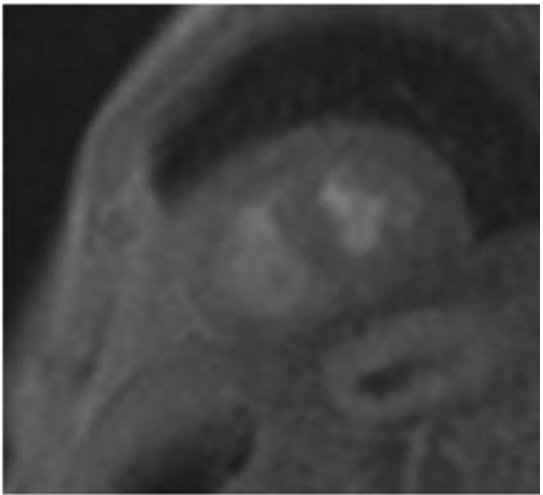

Rest

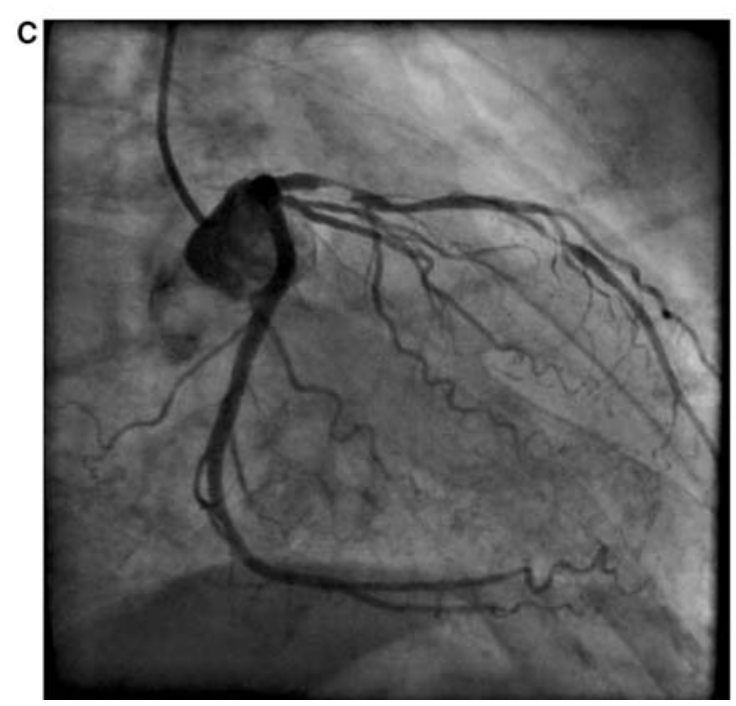

Figure 1. A Polar maps and three-dimensional (3D) ${ }^{201}$ TI-SPECT images with a perfusion defect in the antero-septo-apical region during dipyridamole stress test that widely resolves on the resting perfusion SPECT images ( $S S S=20 ; \mathrm{SRS}=2 ; \mathrm{SDS}=18$ ). B The myocardial perfusion defect seen on polar maps and ${ }^{201}$ TI-SPECT was confirmed by cardiac GRE-MR first-pass perfusion imaging shown here on a short-axis plane during dipyridamole-stimulated hyperemia (left panel) as compared the corresponding short-axis plane at rest (right panel). C Coronary angiography demonstrated a stenosis $>90 \%$ of proximal and middle segment of the left anterior descending artery (LAD). 

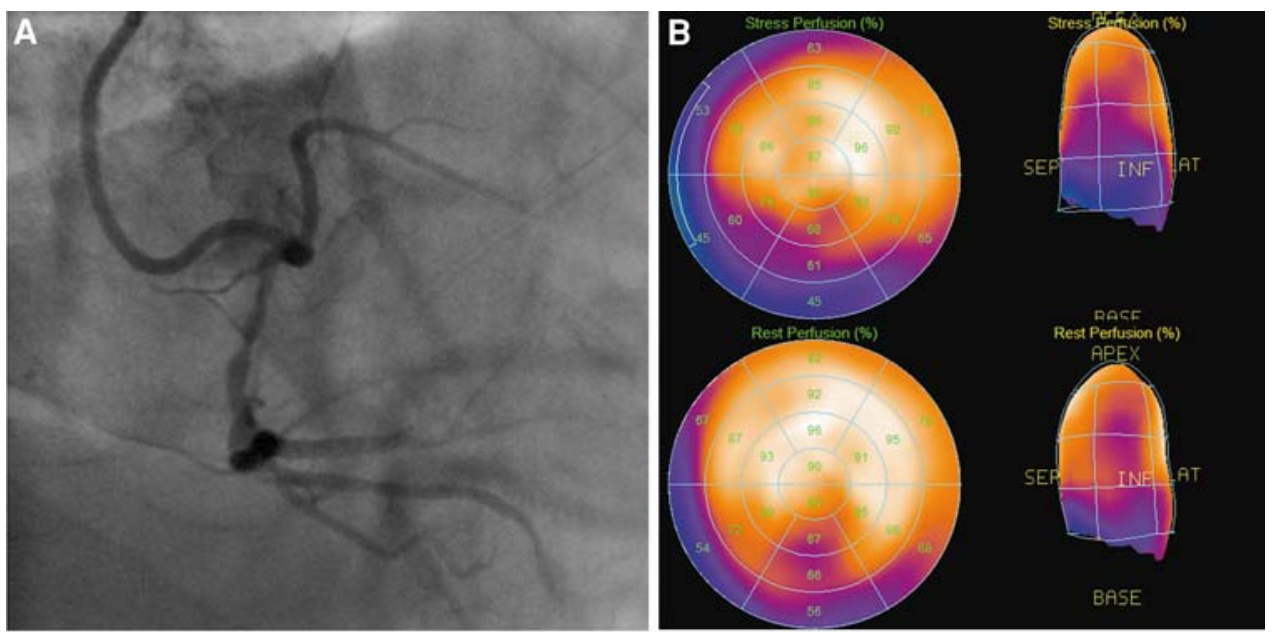

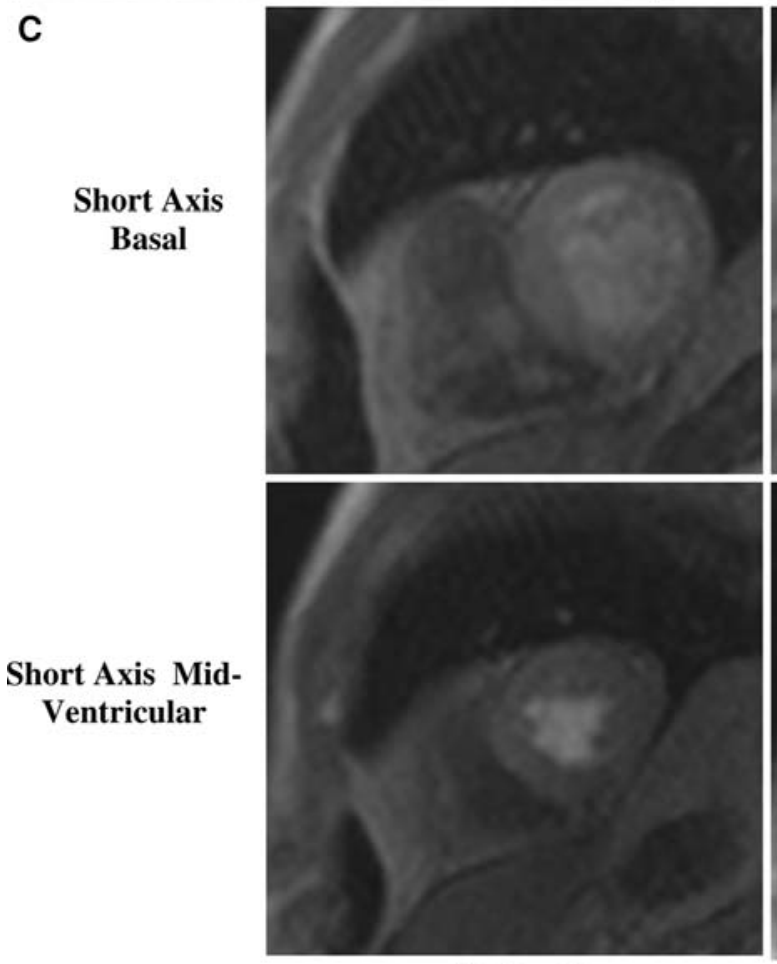

Hyperemia
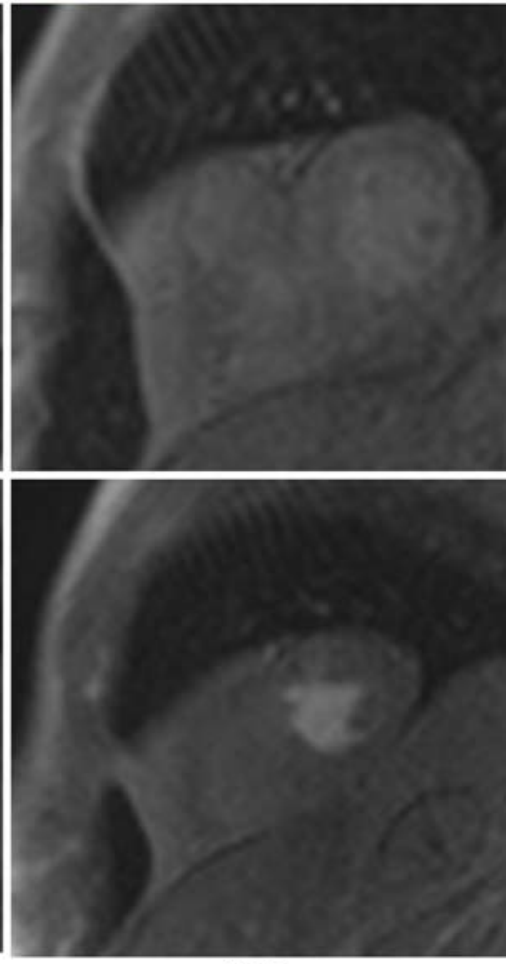

Rest

Figure 2. A Coronary angiography demonstrates a 50\% stenosis of the proximal right coronary artery (RCA) as well as a $90 \%$ stenosis of posterior descending artery (PDA). Subsequently, a ${ }^{201}$ TI-SPECT perfusion study was performed to evaluate the hemodynamic significance of these stenoses. B Polar maps and 3D ${ }^{201}$ TI-SPECT images identify a myocardial perfusion defect in the inferior wall during dipyridamole stimulation that widely resolves on the resting perfusion SPECT images $(\mathrm{SSS}=18, \mathrm{SRS}=7, \mathrm{SDS}=11)$. $\mathbf{C}$ The reversible myocardial perfusion defect denoted on ${ }^{201}$ TI-SPECT was not demonstrated by perfusion cardiac GRE-MR first-pass perfusion imaging on the basal and mid-ventricular short-axis plane during stress (left panel) and rest on corresponding short-axis planes (right panel).

When patients were subgrouped according to the extent of reversible perfusion defects on ${ }^{201}$ TI-SPECT, mild- (SDS: 2-4), moderate- (SDS: 5-8), and severe$($ SDS $>8)$ perfusion defects were also identified by GRE-MRI perfusion analysis in 75\% (6/8), 56\% (9/16), and 100\% (6/6), respectively (Tables 2, 3 and Fig. 4). Table 2 summarizes the head-to-head comparison of ${ }^{201}$ TI-SPECT and GRE-MRI for detecting reversible myocardial perfusion defects in the individual coronary artery. As can be appreciated, some disagreement was 
Table 3. Head-to-head comparison of 201TI-SPECT and GRE-MRI for the detection of reversible myocardial perfusion defects in the individual coronary artery segment

\begin{tabular}{|c|c|c|c|c|c|c|c|c|c|c|c|c|}
\hline \multirow[b]{2}{*}{ Artery } & \multicolumn{3}{|c|}{ Mild ischemia } & \multicolumn{3}{|c|}{ Moderate ischemia } & \multicolumn{3}{|c|}{ Severe ischemia } & \multicolumn{3}{|c|}{ Total ischemia } \\
\hline & SPECT & MRI & $\%$ & SPECT & MRI & $\%$ & SPECT & MRI & $\%$ & SPECT & MRI & $\%$ \\
\hline LAD & 3 & 1 & $33(1 / 3)$ & 4 & 2 & $50(2 / 4)$ & 4 & 4 & $100(4 / 4)$ & 11 & 7 & $65(7 / 11)$ \\
\hline LCX & 3 & 3 & $100(3 / 3)$ & 4 & 3 & $75(3 / 4)$ & - & - & - & 7 & 6 & $86(6 / 7)$ \\
\hline RCA & 2 & 2 & $100(2 / 2)$ & 8 & 4 & $50(4 / 8)$ & 2 & 2 & $100(2 / 2)$ & 12 & 8 & $67(8 / 12)$ \\
\hline Total & 8 & 6 & $75(6 / 8)$ & 16 & 9 & $56(9 / 16)$ & 6 & 6 & $100(6 / 6)$ & 30 & 21 & $70(21 / 30)$ \\
\hline
\end{tabular}

Data in parentheses are numbers used to calculate percentages.

$L A D$, Left anterior descending artery; $L C X$, left circumflex artery; $R C A$, right coronary artery.

Mild ischemia: SDS = 2-4; moderate ischemia: SDS $=5-8$; severe ischemia: SDS $>8$. $\%$ Sensitivity of MRI to detect SPECT defined stress-induced perfusion defects.

(n)

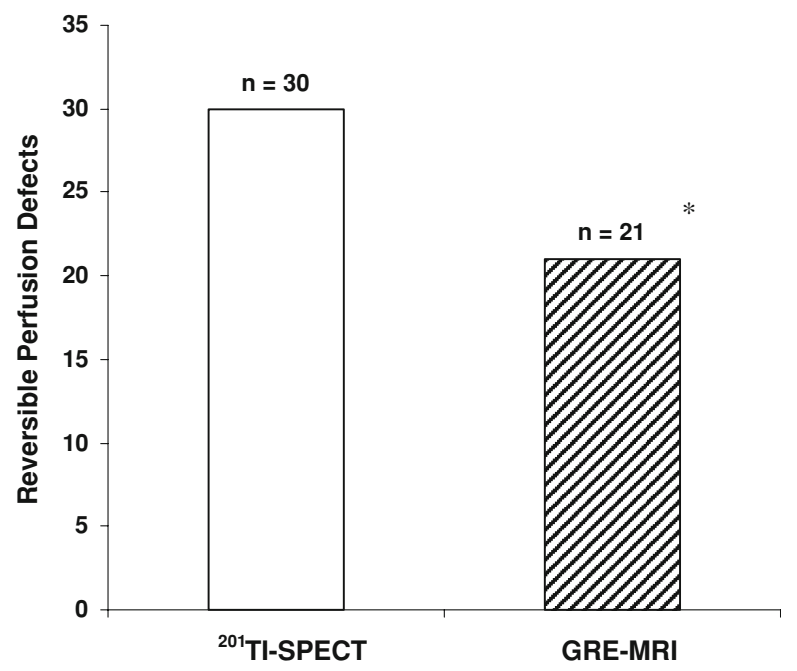

Figure 3. Head-to-head comparison of ${ }^{201}$ TI-SPECT identified myocardial perfusion defects during dipyridamolestimulated hyperemia with GRE-MR first-pass perfusion imaging $\left(* P \leq 0.05\right.$ vs ${ }^{201}$ TI-SPECT).
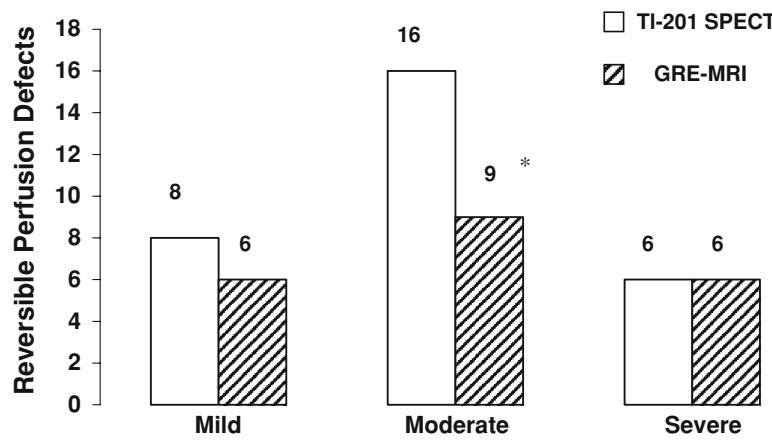

Figure 4. Head-to-head comparison of ${ }^{201}$ TI-SPECT identified mild-, moderate- and severe regional myocardial perfusion defects during dipyridamole-stimulated hyperemia with GREMR first-pass perfusion imaging ( $* P \leq 0.05$ vs ${ }^{201}$ TI-SPECT). (n)

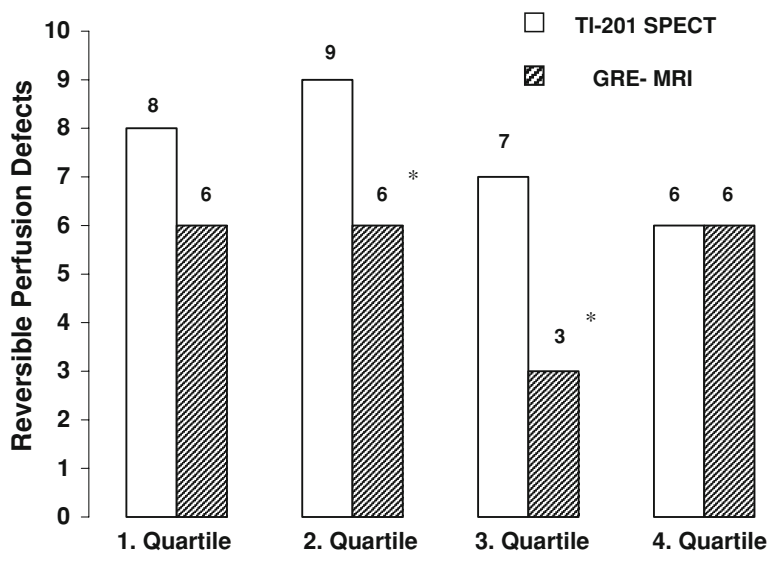

Figure 5. Head-to-head comparison of ${ }^{201}$ TI-SPECT identified myocardial perfusion defects grouped according to quartile analysis of SDS with GRE-MR first-pass perfusion imaging $\left(* P \leq 0.05\right.$ vs ${ }^{201}$ TI-SPECT).

observed in all three coronary territories. In addition, the extent of reversible perfusion defects on ${ }^{201} \mathrm{TI}$-SPECT images was grouped according to quartile analysis with SDS 2-4 (lowest quartile), SDS $>$ 4-6 (lower quartile), SDS $>6-8$ (higher quartile), and $\operatorname{SDS}>8$ (highest quartile). Figure 5 demonstrates that the disagreement between ${ }^{201}$ TI-SPECT and MRI was two in the lowest quartile, three in the lower quartile, and four in the higher quartile, while no disagreement was observed in the highest quartile. Overall, $30 \%(9 / 24)$ of mild-tomoderate regional reversible scintigraphic perfusion defects were not necessarily identified by cardiac MR perfusion imaging, while all six severe reversible perfusion defects were identified by both cardiac perfusion imaging modalities. Differences in hemodynamic conditions during pharmacologically induced hyperemia with dipyridamole during ${ }^{201}$ TI-SPECT and subsequent GRE-MRI, however, may have affected the identification of stress-induced ischemia by GRE-MRI. As 
Table 4. Hemodynamic response to dipyridamole stimulation

\begin{tabular}{|c|c|c|c|c|}
\hline & \multicolumn{2}{|c|}{ SPECT } & \multicolumn{2}{|c|}{ MRI } \\
\hline & Rest & Hyperemia & Rest & Hyperemia \\
\hline HR, beats/min & $71 \pm 10$ & $91 \pm 17$ & $70 \pm 10$ & $86 \pm 19$ \\
\hline SBP, mmHg & $133 \pm 17$ & $139 \pm 20$ & $130 \pm 19$ & $129 \pm 23$ \\
\hline $\mathrm{DBP}, \mathrm{mmHg}$ & $79 \pm 10$ & $79 \pm 11$ & $71 \pm 12$ & $69 \pm 14$ \\
\hline $\mathrm{RPP}, \mathrm{mmHg} \times \min$ & $9523 \pm 1805$ & $12843 \pm 3948$ & $9085 \pm 2045$ & $11347 \pm 4229$ \\
\hline$\triangle \mathrm{RPP}, \mathrm{mmHg} \times \min$ & & $3320 \pm 2143$ & & $2262 \pm 2184$ \\
\hline
\end{tabular}

$H R$, Heart rate; $S B P$, systolic blood pressure; $D B P$, diastolic blood pressure; $R P P$, rate-pressure product $(\mathrm{HR} \times \mathrm{SBP}) ; \triangle R P P$, change of RPP from rest to hyperemia.

$P=0.09$ for $\triangle$ RPP between SPECT vs MRI.

denoted in Table 4, although the stress hemodynamic responses during dipyridamole stimulation statistically did not differ significantly at the time of both myocardial perfusion imaging studies, they tended to be less during GRE-MR perfusion imaging than during the ${ }^{201}$ TISPECT exam. This could have affected, at least in part, the lower detection rate of mild-to-moderate stressinduced regional perfusion defects with GRE-MRI as compared to ${ }^{201}$ TI-SPECT.

\section{DISCUSSION}

In the current study, we aimed to evaluate the diagnostic value of GRE-MR perfusion imaging in the assessment of hemodynamically obstructive CAD in a head-to-head comparison with ${ }^{201}$ TI-SPECT as reference. As it was observed, there was a relatively good concordance of $70 \%$ between cardiac ${ }^{201}$ TI-SPECT and GRE-MRI in the detection of stress-induced regional perfusion defects, while $30 \%$ of mild-to-moderate scintigraphic perfusion defects were not necessarily identified by cardiac GRE-MR perfusion imaging. These observations agree somehow with results of previous investigations in small numbers of patients with suspected $\mathrm{CAD},{ }^{18}$ but extend the latter observations to a larger study population in patients with predominantly known CAD.

The reason for some discordant observation between cardiac ${ }^{201}$ TI-SPECT and GRE-MR imaging in the detection of stress-induced perfusion defects remains uncertain but may be related, at least in part, to differences in the myocardial contrast resolution provided by both cardiac imaging modalities. As regards SPECT perfusion imaging, the myocardial first-pass extraction fraction of ${ }^{201} \mathrm{TI}$ from the blood into the myocardium is as high as $80-85 \% .^{7}$ Beyond this first-pass extraction, recirculation of ${ }^{201} \mathrm{TI}$ in patients allows further extraction of the radiotracer with a further increase in signalto-noise ratio or contrast resolution. Conversely, as coronary flow increases, less ${ }^{201} \mathrm{TI}$ is directly extracted during the capillary transit leading to as so-called "rolloff" at high coronary flow levels. ${ }^{19}$ As a consequence, ${ }^{201} \mathrm{TI}$ uptake is no longer linearly related to myocardial blood flow above $\approx 2.5 \mathrm{~mL} / \mathrm{g} / \mathrm{min}$. The latter drawback in fact may lead to difficulties of ${ }^{201}$ TI-SPECT in the identification of mild regional perfusion differences underlying flow-limiting epicardial lesions during higher hyperemic flow increases above $2.5 \mathrm{~mL} / \mathrm{g} / \mathrm{min}^{7,19}$

As regards the contrast agent gadolinium used for GRE-MR perfusion imaging, it may diffuse rapidly across the capillary membrane into the extracellular space, while it may not enter myocytes with intact cell membranes. ${ }^{20}$ Thus, about $30-50 \%$ of the gadolinium first-pass bolus may enter the myocardial interstitium. It follows then that the myocardial gadolinium concentration is predominantly determined by the intravascular space and thus the coronary flow. First-pass evaluation of the myocardial gadolinium transit is necessary as recirculation and bolus dilation leads to a rapid equilibration of the contrast between the vascular and extravascular space, resulting in a disappearance of a initial myocardial perfusion defect. Due to the latter limitations, the contrast resolution of gadolinium first-pass GRE-MR perfusion imaging is likely to be lower than for myocardial retention of ${ }^{201}$ TI-retention imaged with SPECT. Thus, as observed in the current study, ${ }^{201}$ TI-SPECT with its high myocardial perfusion contrast may be more sensitive to detect more mild-tomoderate stress-induced perfusion defects than gadolinium GRE-MR first-pass imaging. In fact, a survey of the MR first-pass perfusion imaging literature may support these considerations. ${ }^{5}$ According to this, a total of 15 MR perfusion imaging studies with a total of 355 patients were summarized. MR perfusion imaging yielded an average sensitivity of $77 \%$ (ranging from $65 \%$ to $93 \%$ ) and an average specificity of $83 \%$ (range, $60 \%$ to $100 \%$ ). Conversely, the average sensitivity and specificity of ${ }^{201}$ TI-SPECT perfusion imaging during 
dipyridamole stimulation for detecting angiographically significant CAD ( $\geq 50 \%$ stenosis) had been reported of $90 \%$ and $71 \%$, respectively. Sensitivities ranged from $98 \%$ to $76 \%$, with specificities ranging from $42 \%$ to $100 \% .^{7}$ Based on these data, the visual analysis of MR perfusion imaging appears to be less sensitive than SPECT in the identification of flow-limiting epicardial lesion as also observed in the current study, while it may offer a higher specificity. Conversely, MR first-pass imaging with the semiquantitative assessment of an index of myocardial perfusion reserve has been shown to be a promising tool to delineate subendocardial perfusion abnormalities in patients with cardiac syndrome $X$ and normal coronary arteries, ${ }^{21}$ and in a more quantitative analytic approach to even identify mild downstream hemodynamic effects of coronary artery calcification on coronary flows during vasomotor stress in patients with subclinical CAD. ${ }^{22}$ In patients with multivessel $\mathrm{CAD}$, however, the myocardial flow reserve may be reduced in more or less homogenous fashion, ${ }^{23}$ associated with a insufficient regional perfusion disparity to identify underlying flow-limiting epicardial disease. Under such circumstances, therefore, flow-limiting epicardial lesions may remain undetected on conventional visual analysis of relative distributions of the predominantly intravascular gadolinium contrast on MRI or myocardial radiotracer uptake on SPECT images. $^{7,24,25}$ Under such condition, it is possible that the higher contrast resolution of ${ }^{201}$ TI-SPECT may be more sensitive to unravel mild differences in regional myocardial perfusion during hyperemic flow increases that may be missed by the visual analysis of cardiac MR perfusion images. Our observations in patients with predominantly multivessel CAD support such a possibility as nine mild-to-moderate regional reversible perfusion defects as determined by ${ }^{201} \mathrm{TI}$-SPECT were not identified on GRE-MR first-pass perfusion imaging, while none of six severe perfusion defects were missed.

Cardiac SPECT imaging, however, may be significantly affected by artifacts due to photon absorption by the breast, diaphragma, liver, and the thoracic wall leading to false-positive perfusion findings. Consequently, there is a need for attenuation correction by assessing an attenuation map commonly acquired by transmission scanning with external radioactive sources. In particular, the inferior wall has been demonstrated to benefit most from the attenuation correction to avoid false-positive results. ${ }^{13,26}$ As we performed attenuation correction for ${ }^{201}$ TI-SPECT perfusion images, it is unlikely that some perfusion defects during pharmacologic vasodilation were false-positive findings even though we cannot entirely exclude such a possibility. Also, all regional myocardial perfusion defects detected on ${ }^{201}$ TI-SPECT perfusion images were related to epicardial stenosis $>75 \%$ diameter, as evidenced by QCA, that may further emphasize the identification of true perfusion abnormalities on ${ }^{201} \mathrm{TI}$-SPECT in the current study.

Our results agree but also differ from a previous comparative investigation between turbo fast FLASHMR and ${ }^{201}$ TI-SPECT perfusion imaging in 40 patients with suspected CAD. ${ }^{27}$ Applying a morphologic cutoff of $>70 \%$ diameter stenosis of the coronary artery, overall comparable sensitivities and specificities for cardiac turbo-FLASH MRI and ${ }^{201}$ TI-SPECT of $81 \%$ vs $81 \%$ and $68 \%$ vs $63 \%$ were observed, respectively. The relatively lower specificity for ${ }^{201}$ TI-SPECT as compared to MRI may be explained by the fact that ${ }^{201}$ TI-SPECT perfusion images were acquired without attenuation correction. ${ }^{27} \mathrm{An}$ important limitation of the latter study, however, was the heterogeneity in stress testing with bicycle-exercise $(n=26)$ and pharmacologic vasodilation $(n=14)$ for the ${ }^{201}$ TI-SPECT exam. This difference in stress testing in fact may have compromised, at least in part, the comparison of the diagnostic accuracy of both cardiac perfusion imaging modalities in the same patient. Nevertheless, these results were first to have indicated that MR first-pass perfusion imaging may represent an alternative to stress myocardial scintigraphy. This consideration is also supported by more recent findings of the multicentre, multivendor, and randomized MR-impact trial ${ }^{16}$ comparing the role of MR and SPECT perfusion imaging in the detection of flow-limiting epicardial lesions in a study population with relatively high CAD prevalence. Based on a receiver operating characteristic (ROC) analysis, MR perfusion imaging with adenosine stress and an optimal dose of the contrast agent gadolinium $(0.1 \mathrm{mmol} / \mathrm{kg})$ revealed a similar diagnostic performance in the assessment of flow-limiting epicardial lesions of $\geq 50 \%$ diameter stenosis as SPECT using either ${ }^{99 \mathrm{~m}} \mathrm{Tc}$ - or ${ }^{201} \mathrm{TI}$-tracers in a head-to-head comparison. Paradoxically, in the overall study population without a head-to-head comparison and with various doses of gadolinium ranging from 0.01 to $0.1 \mathrm{mmol} / \mathrm{kg}$ for the MRI measurements of myocardial perfusion, MR perfusion imaging apparently proved to be superior to SPECT in the evaluation of flow-limiting epicardial lesions. The reason for this remains uncertain but is likely to be related to the underuse of gated SPECT $(\approx 50 \%)$ and the absence of a proper attenuation correction of SPECT images associated with a higher rate of false-positive findings. In view of the latter limitations of the MR-impact trial, ${ }^{16}$ as the authors also state, future head-to-head comparative trials between MR and SPECT perfusion imaging are needed in patients with low-, intermediate-, and high probability of CAD using a proper methodology and expert reading. 
In general, sensitivities and specificities of myocardial perfusion imaging of CAD depend on several factors such as the patient population studied (i.e., the pretest probability), criteria for $\mathrm{CAD}$, criteria for positive perfusion defects, and imaging methods. ${ }^{28-30}$ Differences in these factors may not necessarily allow a proper comparison of sensitivity and specificity values of different investigations. ${ }^{31,32}$ A morphologic cutoff such as $50 \%$ or $70 \%$ diameter stenosis is a rather poor predictor of hemodynamic downstream effects of coronary stenoses, in particular for intermediate-severity lesions. Thus, comparative studies of different imaging modalities in the assessment of myocardial perfusion in the same patient as performed in the current study may provide more specific and detailed information. It is important to bear in mind, however, that the analysis of ${ }^{201}$ TI-SPECT in the current study was performed quantitatively using the QPS software with a 20-segment model. ${ }^{14}$ According to this, a SSS $\geq 4$ and SDS $\geq 2$ defined the presence of stress-induced myocardial ischemia. Thus, on ${ }^{201}$ TI-SPECT regional myocardial ischemia was defined according to scintigraphic criteria in a quantitative and observer-independent fashion. In the current study, the GRE-MRI analysis of myocardial perfusion was based on a qualitative visual evaluation and, thus, observer-dependent. This may have introduced some observational error in the identification of mild-to-moderate myocardial perfusion defects of cardiac GRE-MR perfusion images. The visual analysis of MR images is known to be hampered sometimes by image artifacts. For example, transient dark rim artifact (DRA) in the subendocardial layer may mimic a hypoperfused area and, thereby, limit the specificity of MR perfusion imaging. The causes of the DRA have been related to so-called Gibbs ringing at the blood-myocardial interface, magnetic susceptibility due to the bolus injection of gadolinium, banding movement artifacts, and partial volume effects between left-ventricular blood pool and the myocardium. ${ }^{33}$ The DRA commonly lasts for a few heart beats and varies temporally as the gadolinium bolus passes through the left-ventricular blood pool. In contrast, a true myocardial perfusion defect tends to be more constant and visible for a longer imaging duration than the DRA. Although an experienced reader can differentiate between DRA and a real perfusion defect by the transient variation of the DRA, it is sometimes difficult to identify true mild-to-moderate perfusion defects which "fill in" rapidly. In this regard, recent investigations ${ }^{34}$ suggest that a fixed DRA (stressrest perfusion defect) on MRI may be unmasked by adding delayed enhancement imaging for the identification or absence of myocardial infarction in the corresponding territory. Such algorithm in fact may increase the diagnostic accuracy in the detection of CAD by incorporating the high specificity of delayed enhancement MRI in a population with intermediate probability of CAD and without prior myocardial infarction. ${ }^{34}$ As the current study population had predominantly known $\mathrm{CAD}$ and previous nontransmural myocardial infarction, the implementation of the delayed enhancement MRI did not aid in the differentiation between DRA and ischemic perfusion defects. In the current study, however, some ${ }^{201}$ TI-SPECT reversible perfusion defects may have been missed by the observer-dependent visual analysis of GRE-MR images due to the difficulties in differentiating between DRA and ischemic perfusion defects. And indeed, four cases with ${ }^{201}$ TI-SPECT defined mild-to-moderate regional perfusion defects were estimated on GRE-MRI as "probably" normal in the presence of some kind of DRA. Another possibility is that if a quantitative and observer-independent analysis had been used for the analysis of the GRE-MR images, these ${ }^{201}$ TI-SPECT defined perfusion defects may have also been identified by GRE-MRI. A previous investigation of cardiac MRI for the detection of $\mathrm{CAD}^{35}$ assessed the diagnostic accuracy of MR first-pass perfusion imaging in the detection of CAD. The visual analysis of the MR perfusion images yielded a sensitivity of $70 \%$, specificity of $78 \%$, and diagnostic accuracy of $74 \%$ when a morphologic significant stenosis was defined as $\geq 75 \%$ diameter. Conversely, using a semiquantitative index of myocardial perfusion reserve in the same study population did lead to an improved sensitivity of $88 \%$, specificity of $90 \%$, and diagnostic accuracy of $89 \%$. Similarly, Schwitter et $\mathrm{al}^{36}$ using a myocardial perfusion reserve index observed a similar sensitivity and specificity of $87 \%$ and $85 \%$, respectively, in detecting coronary artery lesions of $\geq 50 \%$ diameter stenosis. Thus, the semiquantitative assessment of an index of myocardial perfusion reserve or, as it has been reported more recently, ${ }^{37}$ the fractional flow reserve may indeed contribute to improve the diagnostic performance of MR first-pass perfusion imaging in the detection of flow-limiting epicardial lesions. ${ }^{38}$ While such a semiquantitative approach to assess MR first-pass perfusion imaging may contribute to improve the identification flow-limiting epicardial lesions, it is not yet standardized and time-consuming that still limits a more widespread clinical use.

\section{LIMITATIONS}

There are important limitations worthy to be considered in interpreting the current study data. At first, the study population consisted of patients with predominantly with known CAD. The observations therefore could differ among a population with a low- or intermediate risk for CAD. Further comparative investigations are needed to 
evaluate the diagnostic role of MRI first-pass perfusion in the detection of mild-, moderate-, and severe flow-limiting stenosis in patients with suspected CAD. Second, as we only included patients with ${ }^{201}$ TI-SPECT defined ischemia as reference for the MR first-pass perfusion imaging, the current evaluation was biased toward ${ }^{201} \mathrm{TI}-$ SPECT perfusion imaging. According to the latter, this investigation does not allow the commonly comparison of the assessment of sensitivity and specificity values of different cardiac imaging modalities in the detection of $\mathrm{CAD}$ in the clinical setting. Also, the myocardial perfusion studies with ${ }^{201}$ TI-SPECT and GRE-MR first-pass perfusion imaging were not analysed in a blinded manner to each other that could have affected the interpretation of the perfusion images. Third, in view of the relatively small sample size of the study population with ${ }^{201}$ TI-SPECT defined stress-induced perfusion defects, the current investigation does certainly not permit definite conclusions but may add to stimulate larger comparative investigations between ${ }^{201}$ TI-SPECT and MR first-pass perfusion imaging in patients with suspected and known CAD. $^{16}$

\section{CONCLUSIONS}

GRE-MRI first-pass stress perfusion imaging may not identify up to $30 \%$ of mild-to-moderate perfusion defects in a group of preselected patients with predominantly known CAD and abnormal ${ }^{201}$ TI-SPECT studies possibly related to a lower contrast resolution, technical parameters, and/or absence of perfusion quantification.

\section{Acknowledgments}

This work was supported by a clinical research grant of the Department of Internal Medicine of the University Hospitals of Geneva (Switzerland), by a Atherothrombosis Research Fellowship Grant for Dr. G. Vincenti from the European Society of Cardiology (ESC) and the Italian Society of Cardiology (Società Italiana di Cardiologie), and with the support of the Swiss National Science Foundation (SNF grant: PP00B-116901-1).

\section{References}

1. Nesto RW, Kowalchuk GJ. The ischemic cascade: Femporal sequence of hemodynamic, electrocardiographic and symptomatic expressions of ischemia. Am J Cardiol 1987;59(7):23C-30C.

2. Marwick TH. Current status of stress echocardiography for diagnosis and prognostic assessment of coronary artery disease. Coron Artery Dis 1998;9(7):411-26.

3. Geleijnse ML, Elhendy A. Can stress echocardiography compete with perfusion scintigraphy in the detection of coronary artery disease and cardiac risk assessment? Eur $\mathrm{J}$ Echocardiogr 2000;1(1):12-21.
4. Nandalur KR, Dwamena BA, Choudhri AF, Nandalur MR, Carlos RC. Diagnostic performance of stress cardiac magnetic resonance imaging in the detection of coronary artery disease: a meta-analysis. J Am Coll Cardiol 2007;50(14):1343-53.

5. Bax JJ, Van der Wall EE, de Roos A, Poldermans D. Comparison with non-nuclear techniques. In: Zaret BL, Beller GA, editors. Nuclear cardiology - state of the art and future directions. Mosby: Philadelphia; 2005. p. 535-55.

6. Jerosch-Herold M, Kwong RY. Optimal imaging strategies to assess coronary blood flow and risk for patients with coronary artery disease. Curr Opin Cardiol 2008;23(6):599-606.

7. Schindler TH, Schelbert HR. Quantification of myocardial blood flow. In: Dilsizian V, Narula J, Braunwald E, editors. Atlas of nuclear cardiology. Current Medecine LLC; 2006. p. 68-95.

8. Verna E, Ceriani L, Provasoli S, Scotti S, Ghiringhelli S. Larger perfusion defects with exercise compared with dipyridamole SPECT (exercise-dipyridamole mismatch) may reflect differences in epicardial and microvascular coronary dysfunction: When the stressor matters. J Nucl Cardiol 2007;14(6):818-26.

9. Gould KL. Assessing progression or regression of CAD: The role of perfusion imaging. J Nucl Cardiol 2005;12(6):625-38.

10. Schindler TH, Schelbert HR. "Mismatch" in regional myocardial perfusion defects during exercise and pharmacologic vasodilation: A noninvasive marker of epicardial vasomotor dysfunction? J Nucl Cardiol 2007;14(6):769-74.

11. Gould KL, Nakagawa Y, Nakagawa K, Sdringola S, Hess MJ, Haynie M, et al. Frequency and clinical implications of fluid dynamically significant diffuse coronary artery disease manifest as graded, longitudinal, base-to-apex myocardial perfusion abnormalities by noninvasive positron emission tomography. Circulation 2000;101(16):1931-9.

12. Schindler TH, Facta AD, Prior JO, Cadenas J, Zhang XL, Li Y, et al. Structural alterations of the coronary arterial wall are associated with myocardial flow heterogeneity in type 2 diabetes mellitus. Eur J Nucl Med Mol Imaging 2008;36:219-29.

13. Wolak A, Slomka PJ, Fish MB, Lorenzo S, Berman DS, Germano G. Quantitative diagnostic performance of myocardial perfusion SPECT with attenuation correction in women. J Nucl Med 2008;49(6):915-22.

14. Berman DS, Hachamovitch R, Kiat H, Cohen I, Cabico JA, Wang FP, et al. Incremental value of prognostic testing in patients with known or suspected ischemic heart disease: a basis for optimal utilization of exercise technetium- $99 \mathrm{~m}$ sestamibi myocardial perfusion single-photon emission computed tomography. J Am Coll Cardiol 1995;26(3):639-47.

15. Cerqueira MD, Weissman NJ, Dilsizian V, Jacobs AK, Kaul S, Laskey WK, et al. Standardized myocardial segmentation and nomenclature for tomographic imaging of the heart: A statement for healthcare professionals from the Cardiac Imaging Committee of the Council on Clinical Cardiology of the American Heart Association. J Nucl Cardiol 2002;9(2):240-5.

16. Schwitter J, Wacker CM, van Rossum AC, Lombardi M, Al-Saadi $\mathrm{N}$, Ahlstrom H, et al. MR-IMPACT: Comparison of perfusioncardiac magnetic resonance with single-photon emission computed tomography for the detection of coronary artery disease in a multicentre, multivendor, randomized trial. Eur Heart $\mathbf{J}$ 2008;29(4):480-9.

17. Wu E, Judd RM, Vargas JD, Klocke FJ, Bonow RO, Kim RJ. Visualisation of presence, location, and transmural extent of healed Q-wave and non-Q-wave myocardial infarction. Lancet 2001;357(9249):21-8.

18. Bremerich J, Buser P, Bongartz G, Muller-Brand J, Gradel C, Pfisterer $\mathrm{M}$, et al. Noninvasive stress testing of myocardial ischemia: Comparison of GRE-MRI perfusion and wall motion 
analysis to $99 \mathrm{mTc}-\mathrm{MIBI}$-SPECT, relation to coronary angiography. Eur Radiol 1997;7(7):990-5.

19. Glover DK, Ruiz M, Edwards NC, Cunningham M, Simanis JP, Smith WH, et al. Comparison between $201 \mathrm{Tl}$ and $99 \mathrm{mTc}$ sestamibi uptake during adenosine-induced vasodilation as a function of coronary stenosis severity. Circulation 1995;91(3):813-20.

20. Brasch RC. New directions in the development of MR imaging contrast media. Radiology 1992;183(1):1-11.

21. Panting JR, Gatehouse PD, Yang GZ, Grothues F, Firmin DN, Collins $\mathrm{P}$, et al. Abnormal subendocardial perfusion in cardiac syndrome $\mathrm{X}$ detected by cardiovascular magnetic resonance imaging. N Engl J Med 2002;346(25):1948-53.

22. Wang L, Jerosch-Herold M, Jacobs DR Jr, Shahar E, Detrano R, Folsom AR. Coronary artery calcification and myocardial perfusion in asymptomatic adults: The MESA (Multi-Ethnic Study of Atherosclerosis). J Am Coll Cardiol 2006;48(5):1018-26.

23. Di Carli MF, Dorbala S, Hachamovitch R. Integrated cardiac PETCT for the diagnosis and management of CAD. J Nucl Cardiol 2006;13(2):139-44.

24. Beller GA. Underestimation of coronary artery disease with SPECT perfusion imaging. J Nucl Cardiol 2008;15(2):151-3.

25. Dorbala S, Vangala D, Sampson U, Limaye A, Kwong R, Di Carli MF. Value of vasodilator left ventricular ejection fraction reserve in evaluating the magnitude of myocardium at risk and the extent of angiographic coronary artery disease: A $82 \mathrm{Rb}$ PET/CT study. J Nucl Med 2007;48(3):349-58.

26. Slomka PJ, Fish MB, Lorenzo S, Nishina H, Gerlach J, Berman DS, et al. Simplified normal limits and automated quantitative assessment for attenuation-corrected myocardial perfusion SPECT. J Nucl Cardiol 2006;13(5):642-51.

27. Sakuma H, Suzawa N, Ichikawa Y, Makino K, Hirano T, Kitagawa $\mathrm{K}$, et al. Diagnostic accuracy of stress first-pass contrastenhanced myocardial perfusion MRI compared with stress myocardial perfusion scintigraphy. AJR Am J Roentgenol 2005;185(1):95-102.

28. Beller GA. Assessment of new technologies: Surrogate endpoints versus outcomes, and the cost of health care. J Nucl Cardiol 2008;15(3):299-300.
29. Hachamovitch R, Di Carli MF. Methods and limitations of assessing new noninvasive tests: Part II: Outcomes-based validation and reliability assessment of noninvasive testing. Circulation 2008;117(21):2793-801.

30. Hachamovitch R, Di Carli MF. Methods and limitations of assessing new noninvasive tests: Part I: Anatomy-based validation of noninvasive testing. Circulation 2008;117(20):2684-90.

31. Sato A, Hiroe M, Tamura M, Ohigashi H, Nozato T, Hikita H, et al. Quantitative measures of coronary stenosis severity by 64 Slice CT angiography and relation to physiologic significance of perfusion in nonobese patients: comparison with stress myocardial perfusion imaging. J Nucl Med 2008;49(4):564-72.

32. Kern MJ. Coronary physiology revisited: Practical insights from the cardiac catheterization laboratory. Circulation 2000;101(11): 1344-51.

33. Gerber BL, Raman SV, Nayak K, Epstein FH, Ferreira P, Axel L, et al. Myocardial first-pass perfusion cardiovascular magnetic resonance: History, theory, and current state of the art. J Cardiovasc Magn Reson 2008;10(1):18.

34. Klem I, Heitner JF, Shah DJ, Sketch MH Jr, Behar V, Weinsaft J, et al. Improved detection of coronary artery disease by stress perfusion cardiovascular magnetic resonance with the use of delayed enhancement infarction imaging. J Am Coll Cardiol 2006;47(8):1630-8.

35. Nagel E, Klein C, Paetsch I, Hettwer S, Schnackenburg B, Wegscheider K, et al. Magnetic resonance perfusion measurements for the noninvasive detection of coronary artery disease. Circulation 2003;108(4):432-7.

36. Schwitter J, Nanz D, Kneifel S, Bertschinger K, Buchi M, Knusel PR, et al. Assessment of myocardial perfusion in coronary artery disease by magnetic resonance: A comparison with positron emission tomography and coronary angiography. Circulation 2001;103(18):2230-5.

37. Rieber J, Huber A, Erhard I, Mueller S, Schweyer M, Koenig A, et al. Cardiac magnetic resonance perfusion imaging for the functional assessment of coronary artery disease: A comparison with coronary angiography and fractional flow reserve. Eur Heart J 2006;27(12):1465-71.

38. Schwitter J. Myocardial perfusion imaging by cardiac magnetic resonance. J Nucl Cardiol 2006;13(6):841-54. 